\title{
On The Asphericity of a Family of Positive Relative Group Presentations
}

\author{
Suzana Aldwaik and Martin Edjvet \\ School of Mathematical Sciences \\ The University of Nottingham \\ University Park, Nottingham NG7 2RD, UK
}

\begin{abstract}
Excluding four exceptional cases, the asphericity of the relative presentation $\mathcal{P}=\left\langle G, x \mid x^{m} g x h\right\rangle$ for $m \geq 2$ is determined. If $H=\langle g, h\rangle \leq G$, then the exceptional cases occur when $H$ is isomorphic to $C_{5}$ or $C_{6}$.
\end{abstract}

2010 Mathematical subject classification: 20F05, 57M05

Key words: relative group presentations; pictures; asphericity.

\section{Introduction}

A relative group presentation is a presentation of the form $\mathcal{P}=\langle G, \mathbf{x} \mid \mathbf{r}\rangle$, where $G$ is a group and $\mathbf{x}$ is a set disjoint from $G$. Denoting the free group on $\mathbf{x}$ by $\langle\mathbf{x}\rangle, \mathbf{r}$ is a set of cyclically reduced words in the free product $G *\langle\mathbf{x}\rangle$. The group defined by $\mathcal{P}$ is $\hat{G}=$ $G *\langle\mathbf{x}\rangle / N$, where $N$ is the normal closure in $G *\langle\mathbf{x}\rangle$ of $\mathbf{r}$. A relative presentation is said to be aspherical if every spherical picture over it contains a dipole. These notions were defined and studied in [3] where it is shown that if $\mathcal{P}$ is aspherical then group theoretic information about $\hat{G}$ can be deduced.

There has been much interest in determining asphericity of $\mathcal{P}$ particularly when $\mathbf{x}=$ $\{x\}$ and $\mathbf{r}=\{r\}$ both consist of a single element. Indeed, if $r=x^{\varepsilon_{1}} g_{1} \ldots x^{\varepsilon_{k}} g_{k}$ where $g_{i} \in G, \varepsilon_{i}= \pm 1$ and $g_{i}=1$ implies $\varepsilon_{i}+\varepsilon_{i+1} \neq 0(1 \leq i \leq k$, subscripts $\bmod k)$, then the asphericity of $\mathcal{P}$ has been determined (modulo some exceptional cases) when $k \leq 3$ or $r \in\left\{x g_{1} x g_{2} x g_{3} x g_{4}, x g_{1} x g_{2} x g_{3} x^{-1} g_{4}, x g_{1} x g_{2} x g_{3} x g_{4} x g_{5},\left(x g_{1}\right)^{l_{1}}\left(x g_{2}\right)^{l_{2}}\left(x g_{3}\right)^{l_{3}}\left(l_{i}>\right.\right.$ $1,1 \leq i \leq 3)\}$ [1-3] [7-9]. This list includes $x^{m} g x^{-1} h(g, h \in G \backslash\{1\})$ for $m \leq 3$, and when $m \geq 4$ asphericity (modulo exceptional cases) has been determined in [6].

In this paper we consider $x^{m} g x h(g, h \in G \backslash\{1\})$. If $m=2$ then a complete classification of when $\mathcal{P}$ is aspherical has been obtained in [3]. Modulo some exceptions the 
cases $m=3$ and $m=4$ were determined in [2] and [8] respectively. Before stating our main result observe that $x^{m} g x h=1$ if and only if $x^{-m} h^{-1} x^{-1} g^{-1}=1$, and it follows that we can work modulo $g \leftrightarrow h^{-1}$.

We list the following exceptional cases.

(E1) $g=h^{2},|h|=5$ and $m \geq 5$.

(E2) $g \in\left\{h^{2}, h^{3}, h^{4}\right\},|h|=6$ and $m \geq 3$.

Theorem 1.1. Let $\mathcal{P}$ be the relative presentation $\mathcal{P}=\langle G, x| x^{m}$ gxh $\rangle$, where $m \geq 2$, $x \notin G, g, h \in G \backslash\{1\}$. Suppose that none of the conditions in (E1) or (E2) holds. Then $\mathcal{P}$ is aspherical if and only if (modulo $g \leftrightarrow h^{-1}$ ) none of the following holds:

1. $g=h^{ \pm 1}$ has finite order.

2. $g=h^{2}$ has finite order and $m=2$.

3. $g=h^{2},|h|=4$ and $m \geq 3$.

4. $g=h^{2},|h|=5$ and $3 \leq m \leq 4$.

5. $g \in\left\{h^{3}, h^{4}\right\},|h|=6$ and $m=2$.

6. $|g|=2,|h|=3$ and $[g, h]=1$.

7. $\frac{1}{|g|}+\frac{1}{\left|g h^{-1}\right|}+\frac{1}{|h|}>1$, where $\frac{1}{\infty}:=0$.

If $m=2,3,4$ (respectively) then the proof of Theorem 1.1 can be deduced from results in [3], [2], [8] (respectively) apart from two exceptional cases for $m=3$ (E4 and E5 of [2]) which are dealt with here together with the case $m \geq 5$. In Section 2 we discuss the method of the proof where the concept of pictures is needed. In Section 3 some preliminaries results are stated. The proof of Theorem 1.1 is given in Section 4.

\section{Method of Proof}

\subsection{Pictures and Curvature}

The definitions of this subsection are taken from [3]. The reader is referred to [3] and [2] for more details.

A picture $\mathbb{P}$ is a finite collection of pairwise disjoint discs $\left\{D_{1}, \ldots, D_{m}\right\}$ in the interior of a disc $D^{2}$, together with a finite collection of pairwise disjoint simple arcs 
$\left\{\alpha_{1}, \ldots, \alpha_{n}\right\}$ embedded in the closure of $D^{2}-\bigcup_{i=1}^{m} D_{i}$ in such a way that each arc meets $\partial D^{2} \cup \bigcup_{i=1}^{m} D_{i}$ transversely in its end points. The boundary of $\mathbb{P}$ is the circle $\partial D^{2}$, denoted by $\partial \mathbb{P}$. For $1 \leq i \leq m$, the corners of $D_{i}$ are the closures of the connected components of $\partial D_{i}-\bigcup_{j=1}^{n} \alpha_{j}$, where $\partial D_{i}$ is the boundary of $D_{i}$. The regions $\Delta$ of $\mathbb{P}$ are the closures of the connected components of $D^{2}-\left(\bigcup_{i=1}^{m} D_{i} \cup \bigcup_{j=1}^{n} \alpha_{j}\right)$. An inner region of $\mathbb{P}$ is a simply connected region of $\mathbb{P}$ that does not meet $\partial \mathbb{P}$. The picture $\mathbb{P}$ is non-trivial if $m \geq 1$, is connected if $\bigcup_{i=1}^{m} D_{i} \cup \bigcup_{j=1}^{n} \alpha_{j}$ is connected, and is spherical if it is non-trivial and if none of the arcs meets the boundary of $D^{2}$. The number of edges in $\partial \Delta$ is called the degree of the region $\Delta$ and is denoted by $d(\Delta)$. A region of degree $n$ will be called an $n$-region. If $\mathbb{P}$ is a spherical picture, the number of different discs to which a disc $D_{i}$ is connected is called the degree of $D_{i}$, denoted by $d\left(D_{i}\right)$. The discs of a spherical picture $\mathbb{P}$ are also called vertices of $\mathbb{P}$.

Suppose that the picture $\mathbb{P}$ is labelled in the following sense: each $\operatorname{arc} \alpha_{j}$ is equipped with a normal orientation, indicated by a short arrow meeting the arc transversely, and labelled by an element of $\mathbf{x} \cup \mathbf{x}^{-1}$. Each corner of $\mathbb{P}$ is oriented clockwise (with respect to $D^{2}$ ) and labelled by an element of $G$. If $\kappa$ is a corner of a disc $D_{i}$ of $\mathbb{P}$, then $W(\kappa)$ will be the word obtained by reading in a clockwise order the labels on the arcs and corners meeting $\partial D_{i}$ beginning with the label on the first arc we meet as we read the clockwise corner $\kappa$. If we cross an arc labelled $x$ in the direction of its normal orientation, we read $x$, else we read $x^{-1}$.

A picture over $\mathcal{P}$ is a picture $\mathbb{P}$ labelled in such a way the following are satisfied:

1. For each corner $\kappa$ of $\mathbb{P}, W(\kappa) \in \mathbf{r}^{*}$, the set of all cyclic permutations of $\mathbf{r} \cup \mathbf{r}^{-1}$ which begin with a member of $\mathbf{x}$.

2. If $g_{1}, \ldots, g_{l}$ is the sequence of corner labels encountered in anticlockwise traversal of the boundary of an inner region $\Delta$ of $\mathbb{P}$, then the product $g_{1} g_{2} \ldots g_{n}=1$ in $G$. We say that $g_{1} g_{2} \ldots g_{n}$ is the label of $\Delta$, denoted by $l(\Delta)=g_{1} g_{2} \ldots g_{n}$.

A dipole in a labelled picture $\mathbb{P}$ over $\mathcal{P}$ consists of corners $\kappa$ and $\kappa^{\prime}$ of $\mathbb{P}$ together with an arc joining the two corners such that $\kappa$ and $\kappa^{\prime}$ belong to the same region and such that if $W(\kappa)=S g$ where $g \in G$ and $S$ begins and ends with a member of $\mathbf{x} \cup \mathbf{x}^{-1}$, then $W\left(\kappa^{\prime}\right)=S^{-1} g^{-1}$. The picture $\mathbb{P}$ is reduced if it does not contain a dipole. A relative presentation $\mathcal{P}$ is called aspherical if every connected spherical picture over $\mathcal{P}$ contains a dipole. If $\mathcal{P}$ is not aspherical then there is a reduced spherical picture over $\mathcal{P}$. 
The star graph $\mathcal{P}^{\text {st }}$ of a relative presentation $\mathcal{P}$ is a graph whose vertex set is $\mathbf{x} \cup \mathbf{x}^{-1}$ and edge set is $\mathbf{r}^{*}$. For $R \in \mathbf{r}^{*}$, write $R=S g$ where $g \in G$ and $S$ begins and ends with a member of $\mathbf{x} \cup \mathbf{x}^{-1}$. The initial and terminal functions are given as follows: $\iota(R)$ is the first symbol of $S$, and $\tau(R)$ is the inverse of the last symbol of $S$. The labelling function on the edges is defined by $\lambda(R)=g^{-1}$ and is extended to paths in the usual way. A non-empty cyclically reduced cycle (closed path) in $\mathcal{P}^{s t}$ will be called admissible if it has trivial label in $G$. Each inner region of a reduced picture over $\mathcal{P}$ supports an admissible cycle in $\mathcal{P}^{s t}$.

A weight function $\theta$ is a real-valued function on the set of edges of $\mathcal{P}^{s t}$ which satisfies $\theta(S g)=\theta\left(S^{-1} g^{-1}\right)$ where $S g=R \in \mathbf{r}^{*}$. The weight of a closed cycle is the sum of the weights of the constituent edges. A weight function is weakly aspherical if the following conditions are satisfied:

1. Let $R \in \mathbf{r}^{*}$, with $R=x_{1}^{\varepsilon_{1}} g_{1} \ldots x_{n}^{\varepsilon_{n}} g_{n}$. Then

$$
\sum_{i=1}^{n}\left(1-\theta\left(x_{i}^{\varepsilon_{i}} g_{i} \ldots x_{n}^{\varepsilon_{n}} g_{n} x_{1}^{\varepsilon_{1}} g_{1} \ldots x_{i-1}^{\varepsilon_{i-1}} g_{i-1}\right)\right) \geq 2
$$

2. The weight of each admissible cycle in $\mathcal{P}^{s t}$ is at least 2 .

If $\mathcal{P}^{s t}$ admits a weakly aspherical weight function, then $\mathcal{P}$ is aspherical [3] and this method will be used in the proofs.

Another method is curvature distribution (see, for example [7]). Let $\mathbb{P}$ be a reduced spherical picture over $\mathcal{P}$. We proceed as follows. An angle function on $\mathbb{P}$ is a real-valued function on the set of corners of $\mathbb{P}$. Given this, the curvature of a vertex of $\mathbb{P}$ is defined to be $2 \pi$ less the sum of the angles at that vertex. The curvature $c(\Delta)$ of a $k$-gonal region $\Delta$ of $\mathbb{P}$ is the sum of all the angles of the corners of $\Delta$ less $(k-2) \pi$. Our method of associating angles ensures that vertices have zero curvature and it follows from this that $\sum c(\Delta)=4 \pi$ where the sum is taken over all the regions $\Delta$ of $\mathbb{P}$. Assuming that none of conditions 1-7 holds, our strategy will be to show that the positive curvature that exists in $\mathbb{P}$ can be sufficiently compensated by the negative curvature. To this end we locate each $\Delta$ satisfying $c(\Delta)>0$ and distribute $c(\Delta)$ to near regions $\hat{\Delta}$ of $\Delta$. For such regions $\hat{\Delta}$ define $c^{*}(\hat{\Delta})$ to equal $c(\hat{\Delta})$ plus all the positive curvature $\hat{\Delta}$ receives during this distribution procedure. We prove that $c^{*}(\hat{\Delta}) \leq 0$ and, since the total curvature of $\mathbb{P}$ is at most $\sum c^{*}(\hat{\Delta})$, this yields a contradiction which shows that $\mathcal{P}$ is aspherical. 


\subsection{Construction of pictures and Defined angle functions}

For this subsection we assume $g \neq h^{ \pm 1}$. Let $\mathbb{P}$ be a reduced spherical picture over $\mathcal{P}=\left\langle G, x \mid x^{m} g x h\right\rangle$. Then each vertex (disc) in $\mathbb{P}$ has one of the forms given by Figure 2.2.1(i) and (ii); and the the star graph $\mathcal{P}^{s t}$ of $\mathcal{P}$ is given by Figure 2.2.1(iii). Note that when drawing figures the edge arrows shown in Figure 2.2.1 will often be omitted.

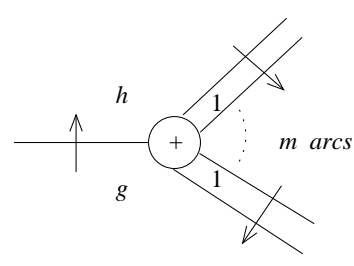

(i)

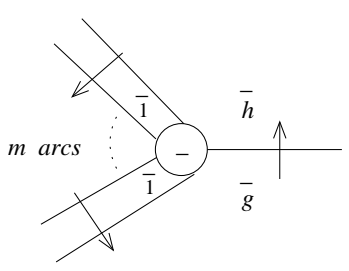

(ii)

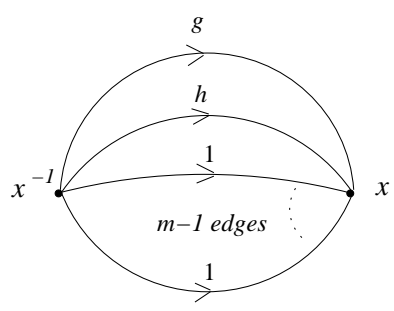

(iii)

Figure 2.2.1: + disc, - disc and $\mathcal{P}^{s t}$.

If there are $k-1$ consecutive regions of degree 2 , then the $k$ arcs in the boundary of these regions constitute a $k$-bond. We will refer to a 1-bond as a single bond. Given that $g \neq h^{ \pm 1}$ there are (up to inversion) only two types of $(m-1)$-bonds in a reduced picture $\mathbb{P}$ (see Figure 2.2.2). For simplicity, in our figures $(m-1)$-bonds will be drawn as bold 2-bonds (see Figure 2.2.2). Note that there are no $m$-bonds or $(m+1)$-bonds in $\mathbb{P}$, indeed a vertex of degree 2 can only occur in a reduced picture if $g=h$ or $g=1$ or $h=1$. Also, for simplicity, the vertex of degree 3 of the form shown in Figure 2.2.3 (i) will be drawn as shown in Figure 2.2.3 (ii), where $m_{1} \geq 2, m_{2} \geq 2$ and $m_{1}+m_{2}=m$.

1)

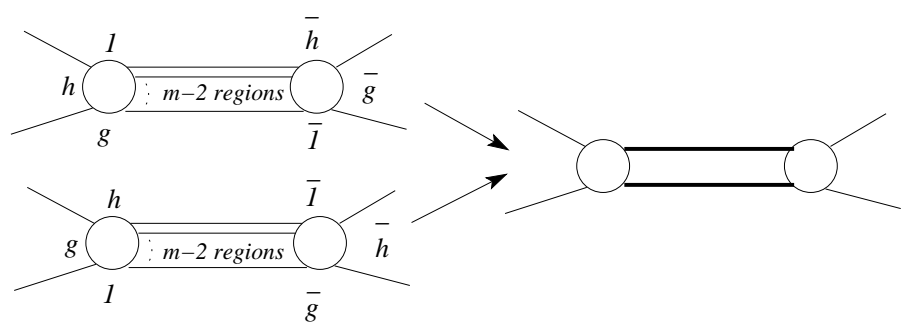

Figure 2.2.2: $(m-1)$-bond. 


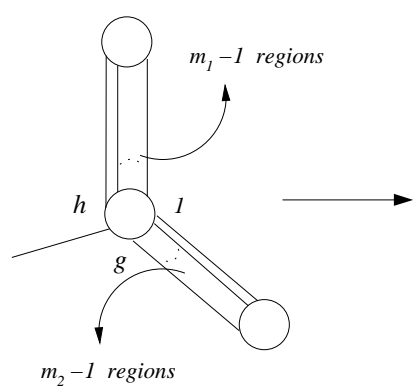

(i)

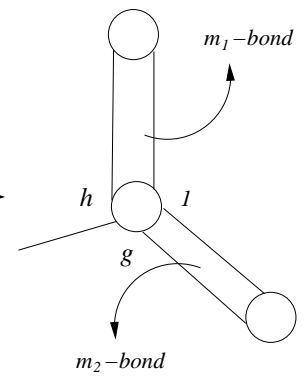

(ii)

Figure 2.2.3.

\section{Remarks 2.1.}

1. Each arc connects $a+$ disc to $a-$ disc, and so each region has even degree.

2. A word $w$ obtained from reading the labels on the edges of a cyclically reduced cycle in $\mathcal{P}^{s t}$ does not contain (up to cyclic permutation and inversion) $g g^{-1}$ or $h h^{-1}$ although it can contain $11^{-1}$ provided different edges in $\mathcal{P}^{\text {st }}$ are used. We will call such words $w$ cyclically reduced .

3. Each region in a reduced spherical picture $\mathbb{P}$ over $\mathcal{P}$ supports a cyclically reduced word in $\{g, h, 1\}$.

There are (up to inversion) three types of vertices of degree 3 and these are shown in Figure 2.2.4.

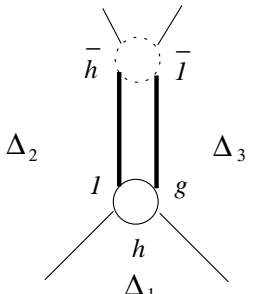

Type 1

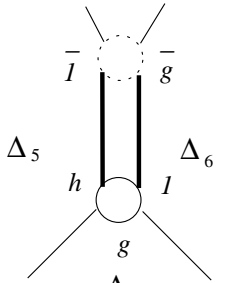

$\Delta_{4}$

Type 2

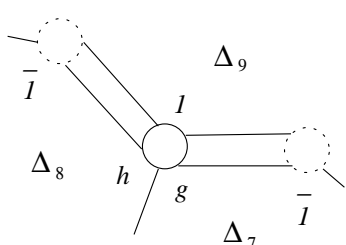

Type 3

Figure 2.2.4.

For the proofs, we define the following angle functions on the vertices $v$ of $\mathbb{P}$. The angle function $\alpha$ is defined as follows. Each corner within a 2-bond has angle zero, while each of the other corners has angle $\frac{2 \pi}{d(v)}$. We will refer to $\alpha$ as the standard angle function. 
The angle function $\alpha_{1}$ is defined as follows. Again, corners within 2-bonds have angle zero. For vertices of degree 3 of Type 1-3, $\alpha_{1}$ is given by Figure 2.2.5. If $d(v)>3$, then each corner in $v$ has angle $\frac{2 \pi}{d(v)}$.

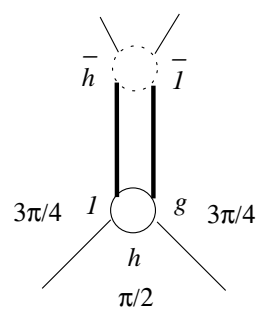

Type 1

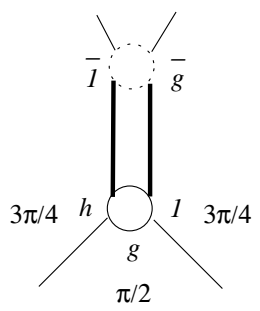

Type 2

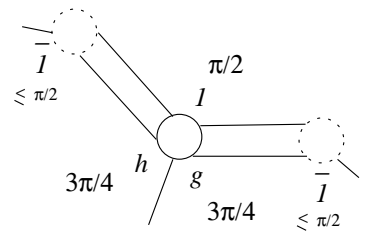

Type 3

Figure 2.2.5: Angle function $\alpha_{1}$ for vertices of degree 3 .

Define an angle function $\alpha_{2}$ on $\mathbb{P}$ as follows. Corners within 2-bonds have angle zero. In vertices of degree 3 , corners labelled by $h^{ \pm 1}$ have angle $\pi$, each of the other two corners has angle $\frac{\pi}{2}$ (see Figure 2.2.6). Corners in vertices of degree $>3$ have angle $\frac{2 \pi}{d(v)}$.

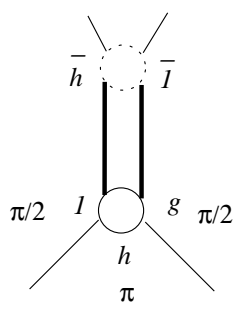

Type 1

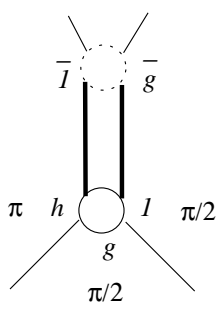

Type 2

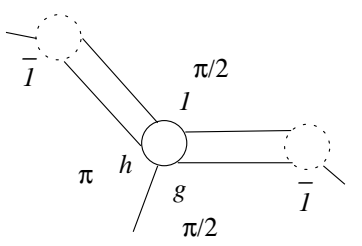

Type 3

Figure 2.2.6: Angle function $\alpha_{2}$ for vertices of degree 3 .

Finally, the angle function $\alpha_{3}$ on $\mathbb{P}$ is given as follows. Corners within 2-bonds have angle zero. For vertices of degree 3 , corners labelled by $1^{ \pm 1}$ have angle $\pi$, each of the other two corners has angle $\frac{\pi}{2}$ (see Figure 2.2.7). Corners in vertices of degree $>3$ have angle $\frac{2 \pi}{d(v)}$. 


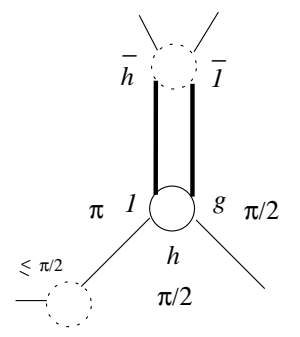

Type 1

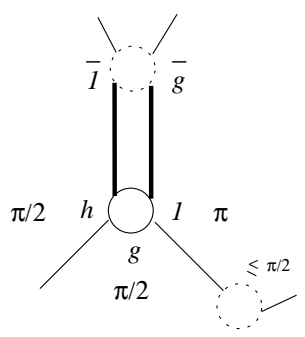

Type 2

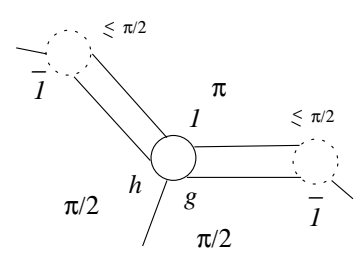

Type 3

Figure 2.2.7: Angle function $\alpha_{3}$ for vertices of degree 3 .

\section{Remarks 2.2.}

1. The corners in each 2-bond have angle 0 in each of the above angle functions. It follows that the curvature of regions of degree 2 is 0 , and so we can treat each $k$-bond as a single bond.

2. By assigning the angle function $\alpha_{1}$ to the corners of $\mathbb{P}$, the following are satisfied:

(i) Since $(2-8) \pi+8 \cdot \frac{3 \pi}{4}=0$, positive regions can only have degree 4 or 6 .

(ii) Both corners adjacent to the $(m-1)$-bond in a boundary of a region have angle $\frac{3 \pi}{4}$; while the two corners adjacent to the $m_{1}$-bond or $m_{2}$-bond in a boundary of a region cannot both have angle $\frac{3 \pi}{4}$ (see Figure 2.2.5).

3. By assigning the angle function $\alpha_{2}$ to the corners of $\mathbb{P}$, the following are satisfied: (i) In any region $\Delta$ of $\mathbb{P}$, there are no consecutive corners with angle $\pi$, else $\mathbb{P}$ is not reduced. Hence, $c(\Delta) \leq(2-n) \pi+\frac{n}{2} \cdot \pi+\frac{n}{2} \cdot \frac{\pi}{2}=\pi\left(\frac{8-n}{4}\right)$ and so positively curved regions can only be 4-regions or 6-regions.

(ii) If $\Delta$ is a positive 4-region, then it has at least one corner labelled by $h^{ \pm 1}$ with angle $\pi$ (otherwise $c(\Delta) \leq-2 \pi+4 \cdot \frac{\pi}{2}=0$ ).

(iii) If $\Delta$ is a positive 6-region, then it contains at least three $h^{ \pm 1}$-corners each with angle $\pi$ (else $\left.c(\Delta) \leq-4 \pi+2 \pi+4 \cdot \frac{\pi}{2}=0\right)$.

4. By assigning the angle function $\alpha_{3}$ to the corners of $\mathbb{P}$, the following are satisfied:

(i) There are no consecutive corners with angle $\pi$ in the boundary of a region $\Delta$ of $\mathbb{P}$ (otherwise $\mathbb{P}$ is not reduced). Thus, $c(\Delta) \leq(2-n) \pi+\frac{n}{2} \cdot \pi+\frac{n}{2} \cdot \frac{\pi}{2}=\pi\left(\frac{8-n}{4}\right)$ and so positive regions can only be 4-regions or 6-regions.

(ii) If $\Delta$ is a positive 4 -region, then it contains at least one corner labelled by $1^{ \pm 1}$ with angle $\pi$ (otherwise $c(\Delta) \leq-2 \pi+4 \cdot \frac{\pi}{2}=0$ ). 
(iii) If $\Delta$ is a positive 6 -region, then it contains three occurrences of $1^{ \pm 1}$-corners each with angle $\pi$ (else $\left.c(\Delta) \leq-4 \pi+2 \pi+4 \cdot \frac{\pi}{2}=0\right)$.

\section{Preliminary Lemmas}

Assume that $m \geq 5$. We first state a series of lemmas followed by their proofs. Recall that we assume $g, h \in G \backslash\{1\}$.

\subsection{Statement of Lemmas}

Lemma 3.1. If $\mathcal{P}$ is not aspherical, then at least one of the following conditions holds:

1. $g=h^{ \pm 1}$;

2. $g=h^{2}$ or $h=g^{2}$;

3. $2 \in\{|g|,|h|\}$;

4. $\left|g h^{-1}\right|=2$ and $3 \in\{|g|,|h|\}$.

Lemma 3.2. If $g=h^{ \pm 1}$, then $\mathcal{P}$ is aspherical if and only if $g$ has infinite order.

Lemma 3.3. Let $g=h^{2}$. If $|h|=4$, then $\mathcal{P}$ is not aspherical, while if $|h|>6$, then $\mathcal{P}$ is aspherical.

Lemma 3.4. If $\frac{1}{|g|}+\frac{1}{\left|g h^{-1}\right|}+\frac{1}{|h|}>1$, then $\mathcal{P}$ is not aspherical.

Lemma 3.5. If $\left|g h^{-1}\right|$ is infinite, then $\mathcal{P}$ is aspherical.

Lemma 3.6. Suppose that $|g|=2$.

1. If $\left|g h^{-1}\right|=2$ and $|h|=\infty$, then $\mathcal{P}$ is aspherical.

2. If $\left|g h^{-1}\right|=3,|h| \geq 6$ and $\mathcal{P}$ is not aspherical, then $g=h^{3}$, in particular $|h|=6$.

3. If $\left|g h^{-1}\right| \geq 4,|h| \geq 4$ and $g \neq h^{2}$, then $\mathcal{P}$ is aspherical.

4. If $\left|g h^{-1}\right| \geq 6$ and $|h|=3$, then $\mathcal{P}$ is not aspherical if and only if $[g, h]=1$.

Lemma 3.7. If $|g|=3,\left|g h^{-1}\right|=2,|h| \geq 6$ and $\mathcal{P}$ is not aspherical, then $g=h^{4}$ and $|h|=6$. 


\subsection{Proof of Lemma 3.1.}

Let $\mathbb{P}$ be a reduced spherical picture over $\mathcal{P}$. It can be assumed without any loss of generality $(\mathbf{A})$ that the number of regions of degree 4 cannot be decreased by bridge moves [5]. Suppose that none of the Conditions 1, 2 or 3 holds.

First assign the standard angle function $\alpha$ to the vertices of $\mathbb{P}$. Since for any $n$ region $\Delta$ in $\mathbb{P}, c(\Delta) \leq \pi\left(\frac{6-n}{3}\right), c(\Delta)>0$ only if $n=4$. A positively curved 4-region $\Delta$ has at least one vertex of degree 3. If $\Delta \in\left\{\Delta_{i}: 1 \leq i \leq 8\right\}$ which are shown in Figure 2.2.4, then at least one corner of $\Delta$ is not labelled by $1^{ \pm 1}$. By considering all cyclically reduced words of length at most 4 in $\left\{g^{ \pm 1}, h^{ \pm 1}\right\}$ (which are compatible with our hypotheses on $g$ and $h$ ), we obtain $l(\Delta)=\left(g h^{-1}\right)^{ \pm 2}$. If $\Delta=\Delta_{9}$ then $l(\Delta)$ gives a contradiction or $\Delta$ is the positive 4-region shown in Figure 3.2.1. Since $m_{1}>B$ a sequence of bridge moves transforms $\Delta$ into a region of degree $>4$ without creating a new region of degree 4 . This contradicts assumption (A) and so by assigning $\alpha$ we obtain $\left|g h^{-1}\right|=2$.

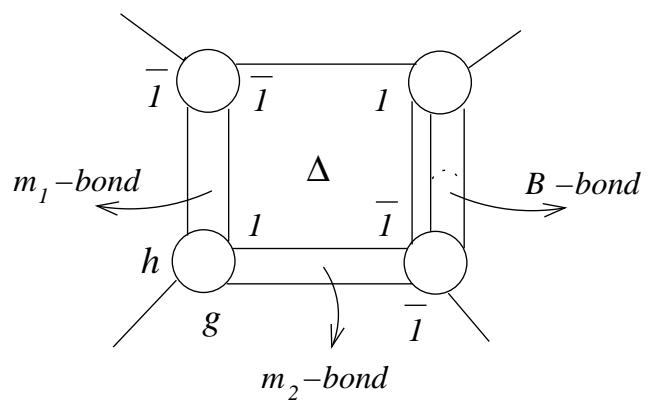

Figure 3.2.1.

Now apply the angle function $\alpha_{1}$. By Remark 2.2.(2)( $\left.i\right)$, positively curved regions can only be 4-regions or 6-regions. A positively curved 4-region $\Delta$ has at least one corner with angle $\frac{3 \pi}{4}$ in its boundary and so $\Delta=\Delta_{i}$ for some $i \in\{2,3,5,6,7,8\}$. This implies that $\Delta$ has at least one corner not labelled by $1^{ \pm 1}$. Also, it implies that $l(\Delta) \neq\left(g h^{-1}\right)^{ \pm 2}$. All other choices contradict our assumptions on $g$ and $h$ and so there are no positive 4 -regions. It follows that $\Delta$ is a 6 -region which contains at least five corners with angle $\frac{3 \pi}{4}$ in its boundary (else, $\left.c(\Delta) \leq(2-6) \pi+4 \cdot \frac{3 \pi}{4}+2 \cdot \frac{\pi}{2}=0\right)$. By Remark 2.2(2)(ii) $\Delta$ contains at least two $(m-1)$-bonds in its boundary and a third bond which is either an $(m-1)$-bond, an $m_{1}$-bond or $m_{2}$-bond. If the $(m-1)$-bonds in the boundary of $\Delta$ are inwardly oriented, then $l(\Delta)=\left(g 1^{-1}\right)^{ \pm 3}$, while if the $(m-1)$ bonds are oriented outward $\Delta$, then $l(\Delta)=\left(h 1^{-1}\right)^{ \pm 3}$. It follows that $\left|g h^{-1}\right|=2$ and $3 \in\{|g|,|h|\}$ which is Condition 4 , as required. 


\subsection{Proof of Lemma 3.2.}

If $g=h$ then $x^{m} g x h=1$ if and only if $x^{m-1}(x g)^{2}=1$. By Lemma 1 in [9], $\mathcal{P}$ is aspherical if and only if $|g|=\infty$.

If $g=h^{-1}$ and $g$ has infinite order, then Lemma 3 in [2] applies to show that $\mathcal{P}$ is aspherical. But $x^{m} g x g^{-1}=1$ and $|g|<\infty$ implies $|x|<\infty$ and by Theorem 1 in [2] $\mathcal{P}$ is not aspherical.

\subsection{Proof of Lemma 3.3.}

Let $g=h^{2}$. For $|h|=4$ there is the sphere shown in Figure 3.4.1. On the other hand if $|h|=k>6$, then the ordinary presentation $\left\langle x, h \mid x^{m} h^{2} x h=1=h^{k}\right\rangle$ is a $\mathrm{C}(4)-\mathrm{T}(4)$ presentation, hence $\mathcal{P}$ is aspherical (for more details see [2]).

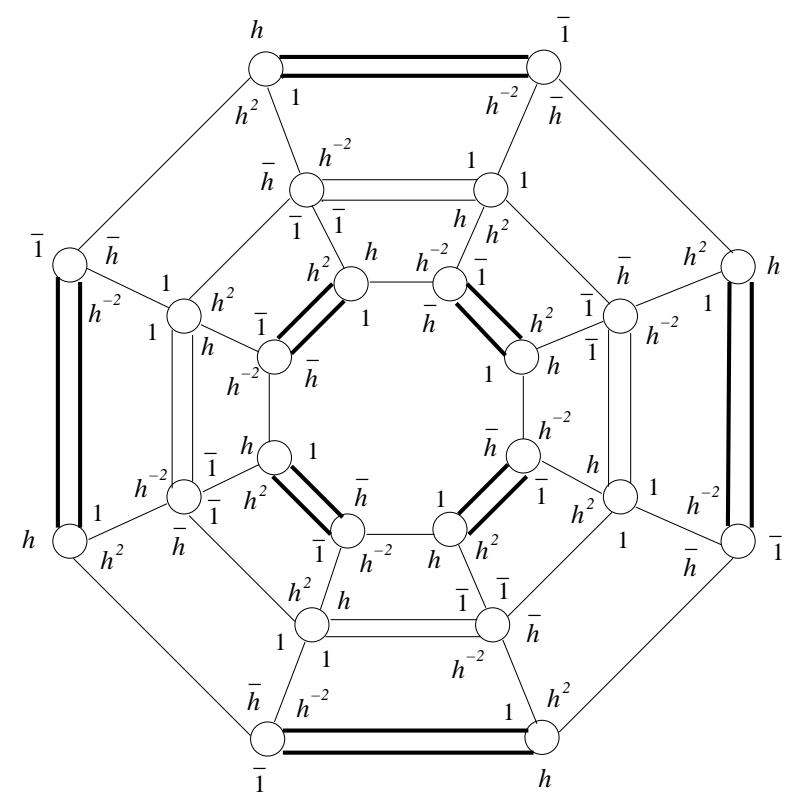

Figure 3.4.1: $g=h^{2}$ and $|h|=4$.

\subsection{Proof of Lemma 3.4.}

If $\frac{1}{|g|}+\frac{1}{\left|g h^{-1}\right|}+\frac{1}{|h|}>1$ then there are spherical pictures $\mathbb{P}$ over $\mathcal{P}$. For example if $\left(|g|,\left|g h^{-1}\right|,|h|\right)=(2,3,4)$ then $\mathbb{P}$ is given by Figure 3.5.1. The other spheres are constructed in a similar way, we omit the details. 


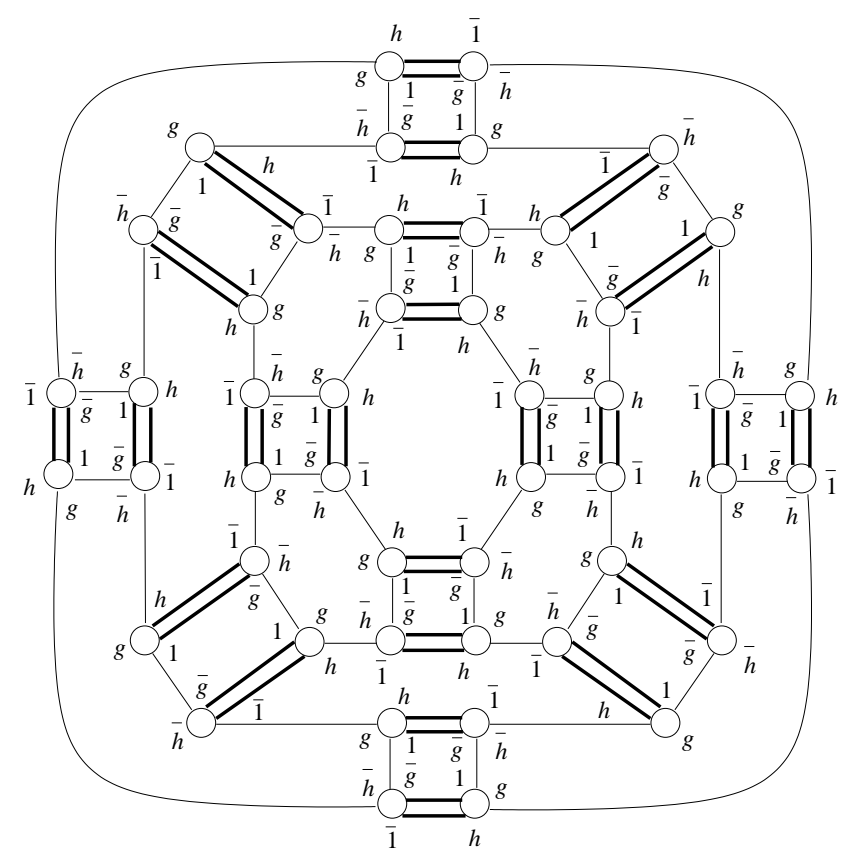

Figure 3.5.1: $\left(|g|,\left|g h^{-1}\right|,|h|\right)=(2,3,4)$.

\subsection{Proof of Lemma 3.5.}

Suppose that $\left|g h^{-1}\right|$ is infinite. If we have a relation of the form $\left(g h^{-1}\right)^{k} g=1$ or $h^{-1}\left(g h^{-1}\right)^{k}=1$ in $G$ then $H=g p\{g, h\}$ is infinite cyclic generated by $g h^{-1}$, and so $\mathcal{P}$ is aspherical by Lemma 3 in [2]. So assume otherwise.

Define the following weight function $\theta$ on $\mathcal{P}^{s t}$ (see Figure 2.2.1(iii)): $\theta\left(e_{g}\right)=0=$ $\theta\left(e_{h}\right)$ and $\theta\left(s_{i}\right)=1$ for $(1 \leq i \leq m-1)$, where $e_{g}, e_{h}, s_{i}(1 \leq i \leq m-1)$ are the edges of $\mathcal{P}^{s t}$ labelled $g, h, 1$ (respectively). Clearly Condition 1 of weakly aspherical weight function is satisfied. The assumptions on $g$ and $h$ imply that each admissible cycle in $\mathcal{P}^{s t}$ must involve at least 2 edges labelled by the identity, and so has weight at least 2 . Therefore $\theta$ is an aspherical weight function which proves that $\mathcal{P}$ is aspherical.

\subsection{Proof of Lemma 3.6(1): Case $(2,2, \infty)$}

In this case, $|g|=2,\left|g h^{-1}\right|=2$ and $|h|=\infty$. Let $\mathbb{P}$ be a reduced spherical picture over $\mathcal{P}$ and assign the angle function $\alpha_{2}$ to $\mathbb{P}$. By Remark $2.2(3)(i)$, positive regions can only be 4-regions or 6-regions. By Remark 2.2 (3)( iii), positive 6-regions involve three occurrences of $h^{ \pm 1}$-corners and each possible label yields a contradiction. By Remark $2.2(3)(i i)$ a positive 4-region must contain $h^{ \pm 1}$ forcing the label $\left(g h^{-1}\right)^{ \pm 2}$. Hence, there are (up to inversion) two types of positive regions as shown in Figure 3.7.1. (Note that 
the maximum possible curvature is always indicated.)

We adopt the notation of [2] and define the following distribution scheme (distributing positive curvature from $\Delta$ to $\hat{\Delta}$ ) which is given in Figure 3.7.1:

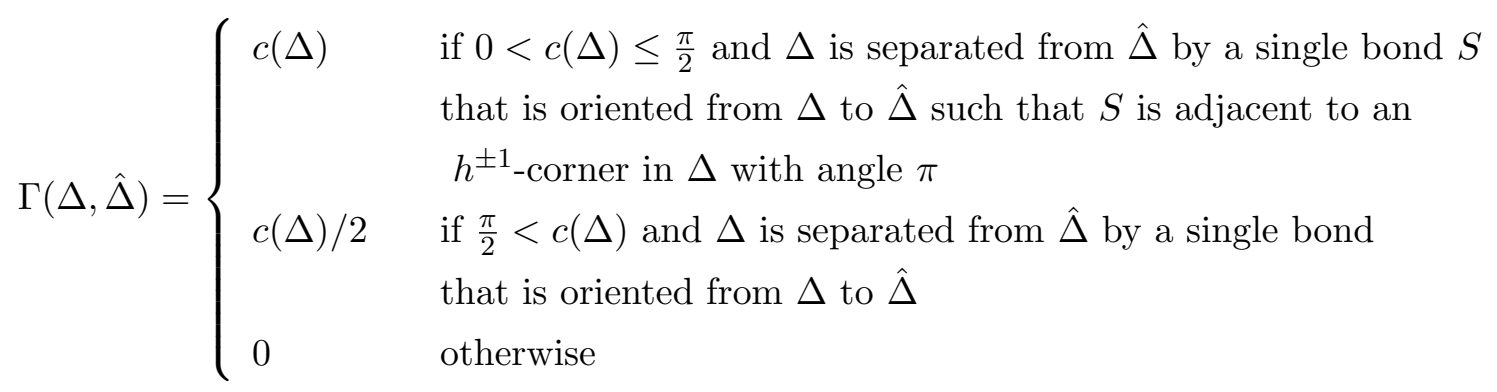
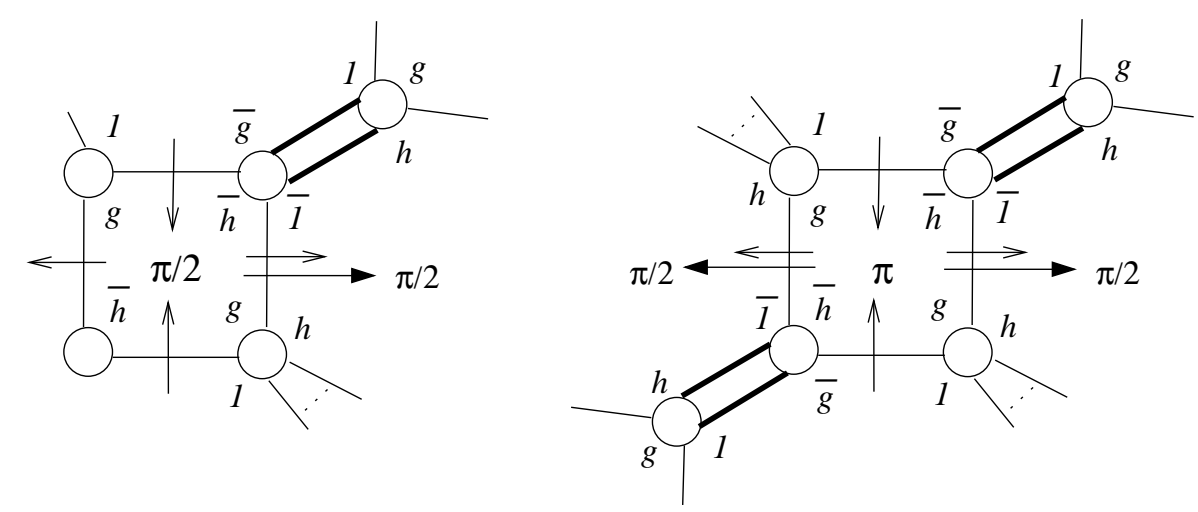

Figure 3.7.1: Positive regions and distribution scheme in Case $(2,2, \infty)$.

Let $\Gamma(\Delta, \hat{\Delta})>0$ and let $r$ be the number of corners of angle $\pi$ in $\hat{\Delta}$. By Remark $2.2(3)(i), r \leq \frac{n}{2}$ where $n=d(\hat{\Delta})$. Set $\Gamma_{2}=\Gamma_{2}(\hat{\Delta})=\left|\left\{\Delta: \Gamma(\Delta, \hat{\Delta})=\frac{\pi}{2}\right\}\right| \leq \frac{n}{2}$ (since $\hat{\Delta}$ receives $\pi / 2$ only across edges that are oriented inwards - see Figure 3.7.1). Then $c^{*}(\hat{\Delta}) \leq(2-n) \pi+r \pi+(n-r) \frac{\pi}{2}+\Gamma_{2} \cdot \frac{\pi}{2}=2 \pi-\frac{\pi}{2}\left(n-r-\Gamma_{2}\right) \leq 2 \pi$. It follows that if $\Gamma_{2} \leq \frac{n}{2}-4$ then $c^{*}(\hat{\Delta}) \leq 0$, so assume otherwise.

If $\Gamma_{2}=\frac{n}{2}$ or $\frac{n}{2}-1$, then (see Figure 3.7.1) the labelling of $\hat{\Delta}$ implies that either $h^{ \pm \frac{n}{2}}=1$ or $g=h^{ \pm \frac{n}{2}}$, contradicting $|h|=\infty$. This leaves $\Gamma_{2}=\frac{n}{2}-3$ and $r=\frac{n}{2}$; or $\Gamma_{2}=\frac{n}{2}-2$ and $r \geq \frac{n}{2}-1$ (otherwise $c^{*}(\hat{\Delta}) \leq 0$ ). First assume that $r=\frac{n}{2}-1$. Then $\Gamma_{2}=\frac{n}{2}-2$ and $c^{*}(\hat{\Delta}) \leq \frac{\pi}{2}$. The fact that $\Gamma_{2}=\frac{n}{2}-2$ means that there are two inwardly oriented edges in $\partial \hat{\Delta}$ across which $\hat{\Delta}$ does not receive $\frac{\pi}{2}$. Figure 3.7.2 $(i)$ shows the first case (consecutive), which forces $l(\hat{\Delta})=h^{\frac{n}{2}-1} w_{1} w_{2} w_{3}$, where $w_{1}, w_{3} \in\left\{1^{-1}, g^{-1}\right\}$ and $w_{2} \in\{1, g, h\}$; and it follows that $|h|<\infty$, a contradiction. The second case is given by Figure 3.7.2 (ii) and $l(\hat{\Delta})=z_{1} h^{\alpha_{1}} z_{2} h^{\alpha_{2}}$, where $z_{1}, z_{2} \in\left\{1^{-1}, g^{-1}\right\}$. If $z_{1}=1^{-1}$ or 
$z_{2}=1^{-1}$ then $|h|=\infty$, a contradiction, so assume otherwise. But if $z_{1}=g^{-1}$ in Figure $3.7 .2(i i)$ then either the $h$-corner in the vertex $v_{1}$ has angle $\leq \frac{\pi}{2}$, or $\Delta_{1}$ contains an $m$-bond in its boundary and so it cannot be either of the positive regions shown in Figure 3.7.1. (i.e $\hat{\Delta}$ does not receive $\frac{\pi}{2}$ from $\Delta_{1}$ ). Either way, $c^{*}(\hat{\Delta})$ will be decreased by $\frac{\pi}{2}$ and so $c^{*}(\hat{\Delta}) \leq 0$.

Now let $r=\frac{n}{2}$ in which case $\Gamma_{2}=\frac{n}{2}-2$ or $\frac{n}{2}-3$ and $c^{*}(\hat{\Delta}) \leq \pi$. Since $g^{2}=\left(g h^{-1}\right)^{2}=1$ it follows that any word in $g$ and $h$ can be rewritten in the form $g^{\alpha_{1}} h^{\alpha_{2}}$. If $g^{ \pm 1}$ appears an odd number of times in $l(\hat{\Delta})$ then $|h|<\infty$. Also, if $g^{ \pm 1}$ occurs at least four times in $l(\hat{\Delta})$ then $\Gamma_{2} \leq \frac{n}{2}-4$, a contradiction, and so $g^{ \pm 1}$ appears exactly twice in $l(\hat{\Delta})$. Since $r=\frac{n}{2}$, each of these two $g^{-1}$-corners is adjacent to two $h$-corners in $\partial \hat{\Delta}$. Thus, arguing as in the case $z_{1}=g^{-1}$ above it follows that $c^{*}(\hat{\Delta}) \leq \pi-2 \cdot \frac{\pi}{2}=0$.

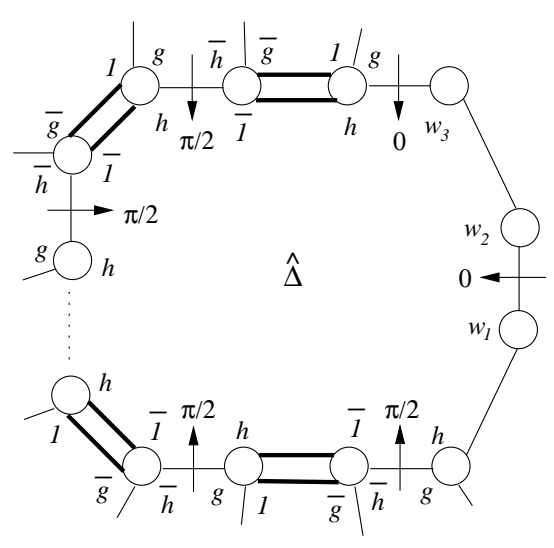

(i) : consecutive

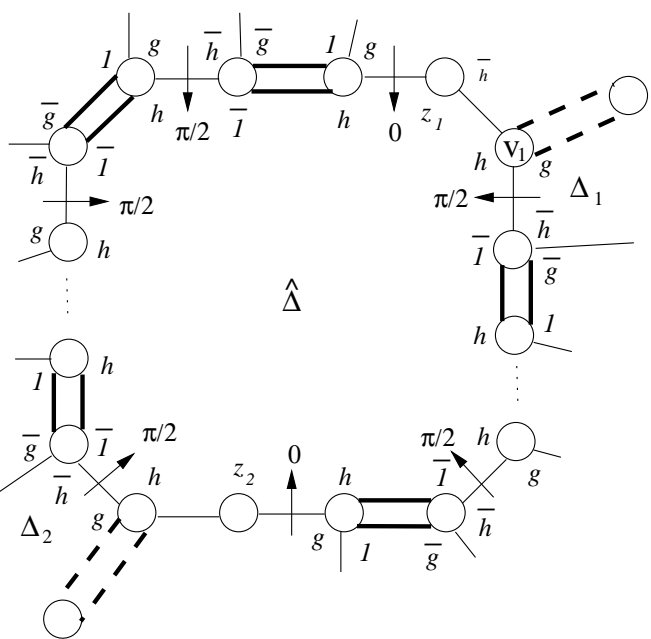

(ii) : non-consecutive

Figure 3.7.2.

\subsection{Proof of Lemma 3.6(2): Case $(2, \overline{3}, \overline{6})$}

Here we assume that $|g|=2,\left|g h^{-1}\right| \geq 3$ and $|h| \geq 6$. Suppose that $\mathcal{P}=\left\langle G, x \mid x^{m} g x h\right\rangle$ is not aspherical. We show that $H=g p\{g, h\}$ is cyclic of order 6 generated by $h$ and $g=h^{3}$. Let $\mathbb{P}$ be a reduced spherical picture over $\mathcal{P}$ to which we assign the angle function $\alpha_{2}$. All possible labels for a positive 4-region give a contradiction since, by Remark $2.2(3)(i i)$, each must involve $h^{ \pm 1}$. For positive 6-regions, by Remark 2.2 (3)(iii), there are three occurrences of $h^{ \pm 1}$ and the only possible labels not yielding a contradiction imply $\left(g h^{-1}\right)^{ \pm 3}=1$ or $g=h^{3}$ (and we are done). Therefore there is (up to inversion) only one positive region which is shown in Figure 3.8.1. 
Apply the following distribution scheme:

$$
\Gamma(\Delta, \hat{\Delta})= \begin{cases}c(\Delta) / 3 & \begin{array}{l}
\text { if } c(\Delta)>0 \text { and } \Delta \text { is separated from } \hat{\Delta} \text { by a single bond } \\
\text { that is oriented from } \Delta \text { to } \hat{\Delta} \\
0
\end{array} \quad \text { otherwise }\end{cases}
$$

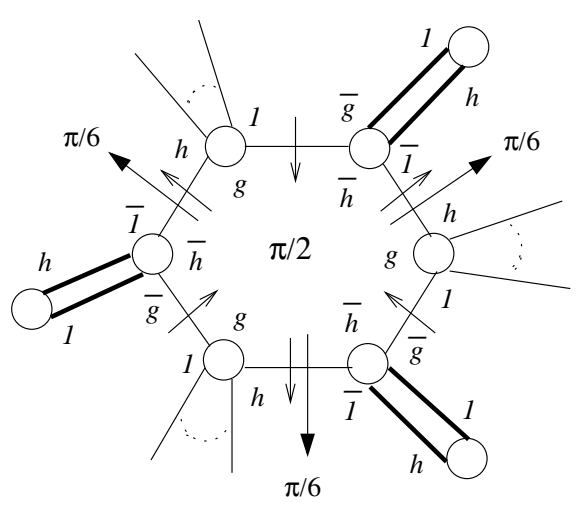

Figure 3.8.1: Positive regions and distribution scheme in Case $(2, \overline{3}, \overline{6})$.

As shown in Figure 3.8.1, if $\Gamma(\Delta, \hat{\Delta})>0$, then $\left(h 1^{-1} h\right)^{ \pm 1}$ is a sublabel of $\hat{\Delta}$. For a fixed region $\hat{\Delta}$ set $\Gamma_{6}(\hat{\Delta})=\left|\left\{\Delta: \Gamma(\Delta, \hat{\Delta})=\frac{\pi}{6}\right\}\right|$.

\section{Remarks 3.8.}

1. Since $\hat{\Delta}$ receives $\pi / 6$ only through edges that are oriented towards $\hat{\Delta}, \Gamma_{6} \leq \frac{n}{2}$.

2. For each $\pi / 6$ that $\hat{\Delta}$ receives, there is an $(m-1)$-bond in the boundary of $\hat{\Delta}$ which gives $\left(h 1^{-1}\right)^{ \pm 1}$ as a sublabel of $\hat{\Delta}$.

3. $l(\hat{\Delta})=h 1^{-1} h w$ and so $d(\hat{\Delta})>6$; since if $d(\hat{\Delta})=6$ then $l(\hat{\Delta})$ yields a contradiction or $g=h^{3}$.

Observe that by Remarks $2.2(3)(i)$ and 3.8.1, $c^{*}(\hat{\Delta}) \leq(2-n) \pi+\frac{n}{2} \cdot \pi+\frac{n}{2} \cdot \frac{\pi}{2}+\frac{n}{2} \cdot \frac{\pi}{6}$ and so $c^{*}(\hat{\Delta})>0$ implies $n<12$.

Let $\hat{\Delta}=(n, r)$ denote a region of degree $n$ with $\Gamma_{6}=r$. We need to check $c^{*}(\hat{\Delta})$ for $\hat{\Delta}=(n, r)=(10,5),(10,4),(10,3),(10,2),(10,1),(8,4),(8,3),(8,2)$ and $(8,1)$. The region $(n, r) \neq(10,5)$ or $(8,4)$ else it gives $h^{ \pm 5}=1$ or $h^{ \pm 4}=1$ (respectively) contradicting $|h| \geq 6$. All possible labels for $\hat{\Delta}=(n, r)=(10,4)$ or $(8,3)$ yields a contradiction. 
For example, $\hat{\Delta}=(8,3)$ gives either $h^{ \pm 4}=1$ or $g=h^{4}$ : the first contradicts $|h| \geq 6$ and the second implies $h=1$. For $\hat{\Delta}=(10, r \leq 3), c^{*}(\hat{\Delta}) \leq(2-10) \pi+5 \pi+5 \cdot \frac{\pi}{2}+3 \cdot \frac{\pi}{6}=0$. Finally, since $(2-8) \pi+3 \pi+5 \cdot \frac{\pi}{2}+2 \cdot \frac{\pi}{6}=-\frac{\pi}{6}<0, c^{*}(\hat{\Delta})>0$ for $\hat{\Delta}=(8, r \leq 2)$ only if it contains 4 corners with angle $\pi$ (up to inversion $\hat{\Delta}$ is shown in Figure 3.8.2), and each possible $l(\hat{\Delta})$ yields a contradiction.

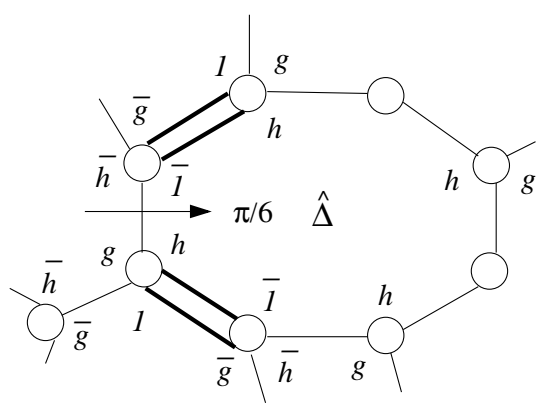

Figure 3.8.2: $\hat{\Delta}=(8, r \leq 2)$ with $c^{*}(\hat{\Delta})>0$.

\subsection{Proof of Lemma 3.6(3): Case $(2, \overline{4}, \overline{4})$}

Here $|g|=2,\left|g h^{-1}\right| \geq 4$ and $|h| \geq 4$. Let $\mathbb{P}$ be a reduced spherical picture over $\mathcal{P}$ and assign the angle function $\alpha_{2}$ to $\mathbb{P}$. By Remark 2.2(3)(i) a positive region $\hat{\Delta}$ can only have degree 4 or 6 . It follows from Remarks $2.2(3)(i i)$ and $(i i i)$ that $l(\hat{\Delta})$ will yield a contradiction. Therefore, in this case $\mathcal{P}$ is aspherical.

\subsection{Proof of Lemma 3.6(4): Case $(2, \overline{6}, 3)$}

If $[g, h]=1$ then $\left|g h^{-1}\right|=6,\left(g h^{-1}\right)^{3}=g$ and $\left(g h^{-1}\right)^{2}=h$. It follows that $\mathcal{P}$ $=\left\langle G, x \mid x^{m} b^{3} x b^{2}, b^{6}\right\rangle$ and this presentation has been shown to be not aspherical by Bogley and Williams [4] (indeed it can be shown that $b$ is conjugate to $x^{m+1}$ ). So it can be assumed that $[g, h] \neq 1$. We prove that $\mathcal{P}=\left\langle G, x \mid x^{m} g x h\right\rangle$ is aspherical. Let $\mathbb{P}$ be a reduced spherical picture over $\mathcal{P}$ with the assumption $(\mathbf{A})$ stated in the proof of Lemma 3.1 and assign the angle function $\alpha_{3}$. By Remark 2.2(4)(i) the degree of a positive region $\Delta$ can only be 4 or 6 . If $\Delta$ is a positive 4 -region with an $h^{ \pm 1}$-corner then $l(\Delta)$ yields a contradiction, so assume otherwise. If now $\Delta$ has a $g^{ \pm 1}$ corner then $l(\Delta)$ yields the 4-regions shown in Figure 3.10.1. This leaves $l(\Delta)=11^{-1} 11^{-1}$ which contradicts (A) as in the proof of Lemma 3.1.

If $\Delta$ is a 6 -region, then either there is a contradiction or $l(\Delta) \in\left\{1^{-1} 11^{-1} 11^{-1} 1\right.$, $\left.1^{-1} 11^{-1} g 1^{-1} g, 1^{-1} h 1^{-1} h 1^{-1} h\right\}$. The first two cannot be positive, while the last gives the positive 6-region shown in Figure 3.10.1. 
Define the following distribution scheme which is given in Figure 3.10.1:

$$
\Gamma(\Delta, \hat{\Delta})= \begin{cases}c(\Delta) / 2 & \text { if } c(\Delta)=\pi \text { and } \Delta \text { is separated from } \hat{\Delta} \text { by a single bond } \\ & \text { that is oriented from } \Delta \text { to } \hat{\Delta} \\ c(\Delta) & \text { if } 0<c(\Delta) \leq \frac{\pi}{2}, \Delta \text { is separated from } \hat{\Delta} \text { by a single bond } S \\ & \text { that is oriented from } \Delta \text { to } \hat{\Delta} \text { and } S \text { is adjacent to a 1-corner } \\ & \text { in } \Delta \text { with angle } \pi \\ \pi / 6 & \text { if } c(\Delta)=\frac{\pi}{2} \text { and } \Delta \text { is separated from } \hat{\Delta} \text { by a single bond } \\ & \text { that is oriented from } \hat{\Delta} \text { to } \Delta \\ & \text { otherwise }\end{cases}
$$
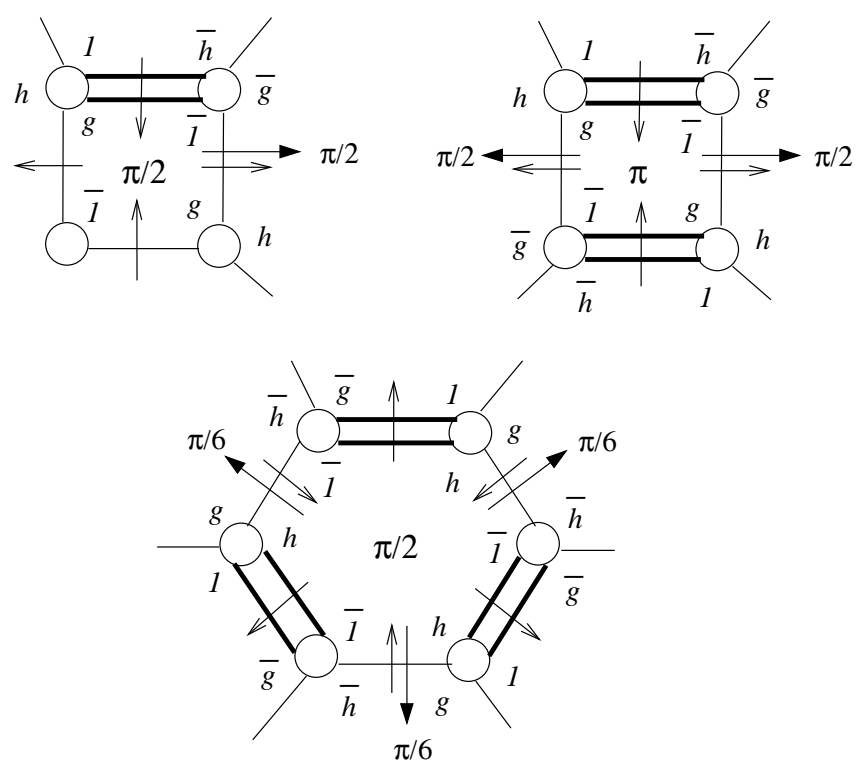

Figure 3.10.1: Positive regions and distribution scheme in Case $(2, \overline{6}, 3)$.

Let $r$ be the number of corners of angle $\pi$ in $\Delta$. Then $r \leq \frac{n}{2}$ (by Remark 2.2(4)(i)). Let $s$ denote the number of pairs $\left(\frac{\pi}{2}, \frac{\pi}{6}\right)$ or $\left(\frac{\pi}{6}, \frac{\pi}{2}\right)$ such that $\hat{\Delta}$ receives $\frac{\pi}{2}$ and $\frac{\pi}{6}$ across adjacent edges in $\partial \hat{\Delta}$, with the understanding that each $\frac{\pi}{2}$ and $\frac{\pi}{6}$ that $\hat{\Delta}$ receives appears at most once in these pairs. Denote the remaining number of $\frac{\pi}{2}$ that $\hat{\Delta}$ receives by $s_{1}$. Also, let $s_{2}$ denote the remaining number of $\frac{\pi}{6}$ that $\hat{\Delta}$ receives. As an example to show how to get the values $s, s_{1}$ and $s_{2}$ see Figure 3.10.2. 


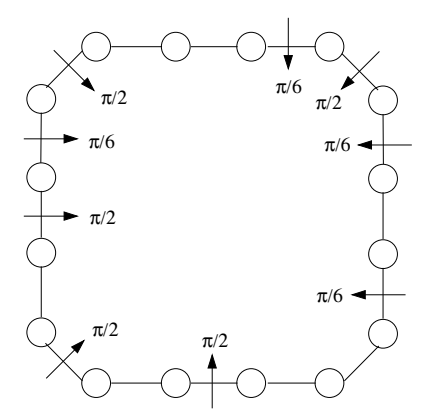

Figure 3.10.2: $n=16, s=2, s_{1}=3, s_{2}=2$.

\section{Remarks 3.9.}

1. As shown in Figure 3.10.1, $l(\hat{\Delta}) \in\left\{h g^{-1} w, h^{-1} g w\right\} \Rightarrow d(\hat{\Delta})>6$ for otherwise $l(\hat{\Delta})$ yields a contradiction.

2. $r \leq \frac{n}{2}-\left(s+s_{1}+s_{2}\right)$.

3. $s+s_{2} \leq \frac{n}{2}$.

Let $\hat{\Delta}$ be a region such that $c^{*}(\hat{\Delta})>0$. Then $c^{*}(\hat{\Delta}) \leq(2-n) \pi+\left[\frac{n}{2}-\left(s+s_{1}+\right.\right.$ $\left.\left.s_{2}\right)\right] \pi+\left(\frac{n}{2}+s+s_{1}+s_{2}\right) \frac{\pi}{2}+s\left(\frac{1}{2}+\frac{1}{6}\right) \pi+s_{1} \cdot \frac{\pi}{2}+s_{2} \cdot \frac{\pi}{6}=\frac{\pi}{12}\left(24-3 n+2 s-4 s_{2}\right)$, and so $c^{*}(\hat{\Delta})>0$ implies $24-3 n+2 s-4 s_{2}>0 \Rightarrow 3 n<24-4 s_{2}+2 s \leq 24-4 s_{2}+2\left(\frac{n}{2}-s_{2}\right)=$ $24-6 s_{2}+n \Rightarrow n<12$.

Let $n=10$. Then $c^{*}(\hat{\Delta})>0 \Rightarrow 24-3(10)+2 s>4 s_{2} \geq 0 \Rightarrow s>3$. If $s=4$ or 5 , then either $l(\hat{\Delta})=\left(g^{-1} h\right)^{4} g^{-1} 1$ which contradicts $[g, h] \neq 1$ or $l(\hat{\Delta})=\left(g^{-1} h\right)^{5}$ which contradicts $\left|g h^{-1}\right| \geq 6$. This leaves $n=8$. But checking the possible labels shows that $l(\hat{\Delta})=h g^{-1} 1 g^{-1} h 1^{-1} h 1^{-1} \Rightarrow c^{*}(\hat{\Delta}) \leq-\frac{\pi}{3}$ (see Figure 3.10.3).

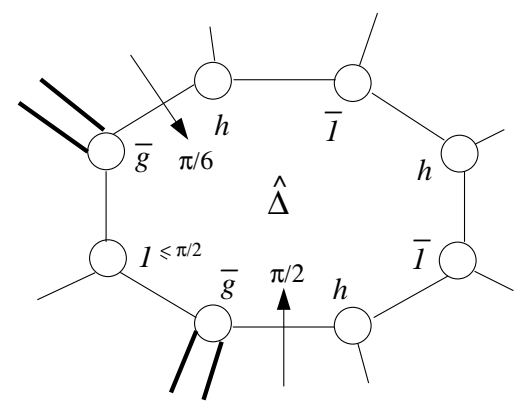

Figure 3.10.3: $n=8$.

\subsection{Proof of Lemma 3.7: Case $(3,2, \overline{6})$}

Here, we assume that $|g|=3,\left|g h^{-1}\right|=2$ and $|h| \geq 6$. Let $\mathbb{P}$ be a reduced spherical picture over $\mathcal{P}$ and assign the angle function $\alpha_{1}$ to $\mathbb{P}$. Observe that if $c(\hat{\Delta})>0$, then 
$l(\hat{\Delta}) \in\left\{1^{-1} g w, h 1^{-1} w\right\}$ (see Figure 2.2.6). It follows that all positively curved regions are shown in Figure 3.11.1.

Define the following distribution scheme which is given in Figure 3.11.1:

$\Gamma(\Delta, \hat{\Delta})= \begin{cases}\pi / 6 & \text { if } c(\Delta)=\frac{\pi}{2} \text { and } \Delta \text { is separated from } \hat{\Delta} \text { by an }(m-1) \text {-bond } \\ c(\Delta) / 2 & \text { if } 0<c(\Delta) \leq \frac{\pi}{4} \text { and } \Delta \text { is separated from } \hat{\Delta} \text { by an }(m-1) \text {-bond } \\ 0 & \text { otherwise }\end{cases}$
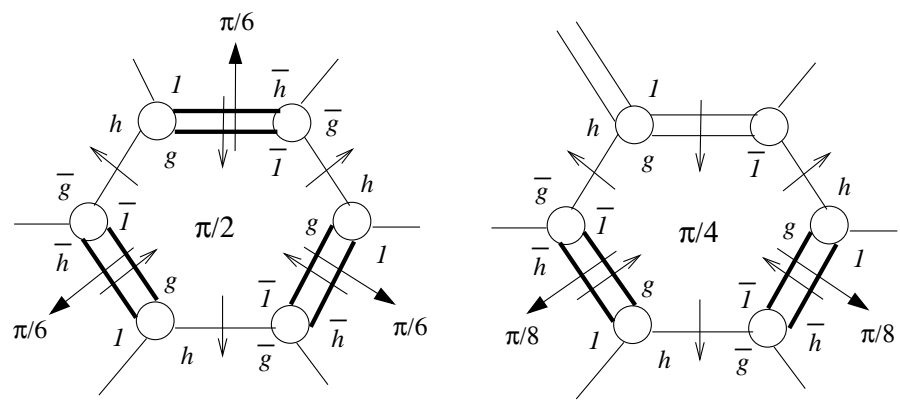

Figure 3.11.1: Positive regions and distribution scheme in Case $(3,2, \overline{6})$.

For a fixed region $\hat{\Delta}$ again set $\Gamma_{6}(\hat{\Delta})=\left|\left\{\Delta: \Gamma(\Delta, \hat{\Delta})=\frac{\pi}{6}\right\}\right|$.

\section{Remarks 3.10.}

1. The region $\hat{\Delta}$ receives each $\pi / 6$ through an $(m-1)$-bond in its boundary which gives $\left(1 h^{-1}\right)^{ \pm 1}$ as a sublabel of $\hat{\Delta}$.

2. $\hat{\Delta}$ receives $\pi / 6$ only through edges that are oriented outwards $\hat{\Delta}$, and so $\hat{\Delta}$ does not receive $\pi / 6$ through consecutive edges in its boundary $\left(\Gamma_{6} \leq \frac{n}{2}\right)$. Also, for each $\pi / 6$ that $\hat{\Delta}$ receives, there are two corners in $\hat{\Delta}$ with angle $\frac{3 \pi}{4}$. Therefore, $\Gamma_{6} \leq \frac{r}{2}$, where $r$ is the number of corners with angle $\frac{3 \pi}{4}$ in the boundary of $\hat{\Delta}$.

3. As shown in Figure 3.11.1, $l(\hat{\Delta})=1 h^{-1} w$, which implies that $d(\hat{\Delta})>4$ for otherwise $l(\hat{\Delta})$ yields a contradiction.

By using $\Gamma_{6} \leq \frac{r}{2}, c^{*}(\hat{\Delta}) \leq(2-n) \pi+r \cdot \frac{3 \pi}{4}+(n-r) \cdot \frac{\pi}{2}+\frac{r}{2} \cdot \frac{\pi}{6}$, and so $c^{*}(\hat{\Delta})>0$ $\Rightarrow 2 r>3 n-12$. Since $r \leq n$, this implies that $n<12$. 
Let $\hat{\Delta}=(n, r)$ denote a region of degree $n$ with $r$ corners of angle $\frac{3 \pi}{4}$ and assume that $c^{*}(\hat{\Delta})>0$. Since $2 r>3 n-12$ it follows that if $n=10$ then $r=10$; if $n=8$ then $r=7$ or 8 ; and if $n=6$ then $r=4,5$ or 6 . If $(n, r)=(10,10)$ or $(8,8)$ then $l(\hat{\Delta})$ implies that $h^{5}=1$ or $h^{4}=1$ contradicting $|h| \geq 6$. If $(n, r)=(8,7)$ then $\hat{\Delta}$ is given by Figure $3.11 .2(i)$ and $l(\hat{\Delta})$ implies either $h^{4}=1$ or $g=h^{4}$ which is $(\mathbf{E 2})$. This leaves $d(\hat{\Delta})=6$ and checking shows that $l(\hat{\Delta})=1 h^{-1} g h^{-1} g 1^{-1}$ as in Figure 3.11.2 $(i i)$, otherwise there is a contradiction or condition (E2) occurs. But observe that if $r>3$ in Figure 3.11.2 (ii) then $r=4$ and since the $\hat{\Delta}$ corners of vertices $u$ and $v$ cannot have angle $\frac{3 \pi}{4}$, this forces $x=h^{-1}$, a contradiction which completes the proof.

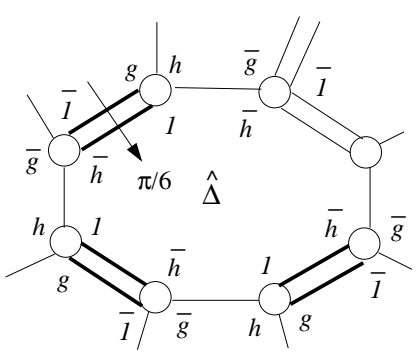

(i)

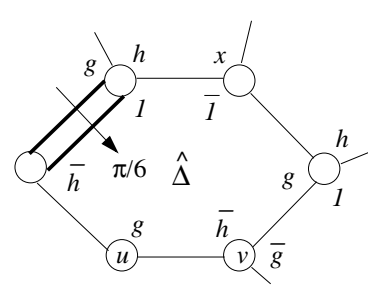

(ii)

Figure 3.11.2.

\section{Proof of Theorem 1.1}

As mentioned in the introduction, the proof of Theorem 1.1 has been done previously for $m=2,3$ and 4 except for the exceptional cases E4 and E5 of [2]. But by following the proof of Lemma 3.6(3), Lemma 8(3) in [2] can be amended as follows: if $|g|=2$, $\left|g h^{-1}\right| \geq 4,|h| \geq 4$ and $g \neq h^{2}$, then $\mathcal{P}$ is aspherical even if $[g, h]=1$; and so in these two cases $\mathcal{P}$ is also aspherical. So it can be assumed that $m \geq 5$. The 'only if' direction of Theorem 1.1 follows from Lemmas 3.2, 3.3, 3.4 and 3.6(4). For the rest of the proof we assume that none of the Conditions (1)-(7) of Theorem 1.1 is satisfied. We show that either $\mathcal{P}$ is aspherical or exceptional.

If none of the conditions of Lemma 3.1 holds, then $\mathcal{P}$ is aspherical. Assume that Condition 1 of Lemma 3.1 holds. Then $|g|=\infty$ (since Condition 1 of Theorem 1.1 does not hold), and so $\mathcal{P}$ is aspherical by Lemma 3.2. So assume from now on that $g \neq h^{ \pm 1}$.

If Condition 2 of Lemma 3.1 holds, then it can be assumed without any loss that $g=h^{2}$. Then $|h| \geq 5$ (by the negation of Condition 3 of Theorem 1.1). If $|h| \in\{5,6\}$ then $\mathcal{P}$ is exceptional of type $(\mathbf{E} 1)$ or $\left(\mathbf{E} 2, g=h^{2}\right)$; and if $|h| \geq 7$, then $\mathcal{P}$ is aspherical by Lemma 3.3. So assume from now on that $g \neq h^{2}$. 
If Condition 3 of Lemma 3.1 holds, then it can be assumed without any loss that $|g|=2$. Since $g \neq h,\left|g h^{-1}\right| \geq 2$. If $\left|g h^{-1}\right|=2$ then $|h|=\infty$ (Condition 7 of Theorem 1.1) and it follows that $\mathcal{P}$ is aspherical by Lemma 3.6(1). If $\left|g h^{-1}\right|=3$, then $|h| \geq 6$ (Condition 7 of Theorem 1.1). By Lemma 3.6(2), $\mathcal{P}$ is aspherical if $g \neq h^{3}$, while if $g=h^{3}$ then $\mathcal{P}$ is exceptional of type $\left(\mathbf{E 2}, g=h^{3}\right)$. If $\left|g h^{-1}\right|=4$ or 5 then $|h| \geq 4$ (Condition 7 of Theorem 1.1), and so $\mathcal{P}$ is aspherical by Lemma 3.6(3). Now suppose that $\left|g h^{-1}\right| \geq 6$. By Lemma 3.5, if $\left|g h^{-1}\right|=\infty$ then $\mathcal{P}$ is aspherical, so assume otherwise. Then $|h| \geq 3$ (Condition 7 of Theorem 1.1). If $|h|=3$ then $[g, h] \neq 1$, otherwise Condition 6 of Theorem 1.1 holds, and so $\mathcal{P}$ is aspherical by Lemma 3.6(4). If $|h| \geq 4$, then $\mathcal{P}$ is aspherical by Lemma 3.6(3).

Finally, if Condition 4 of Lemma 3.1 is satisfied then it can be assumed without loss that $|g|=3$ and $\left|g h^{-1}\right|=2$. Hence $|h| \geq 6$ (else, Condition 7 of Theorem 1.1 applies). If $g=h^{4}$ then $\mathcal{P}$ is exceptional of type $\left(\mathbf{E 2}, g=h^{4}\right)$; otherwise $\mathcal{P}$ is aspherical by Lemma 3.7.

\section{References}

[1] A.Ahmad, M. Al-Mulla, and M. Edjvet, "Asphericity of length four relative group presentations", preprint (2013).

[2] Y. G. Baik, W. A. Bogley, and S. J. Pride, "On the asphericity of length four relative group presentations", Internat. J. Algebra Comput. 7 (1997), 227-312.

[3] W. A. Bogley, and S. J. Pride, "Aspherical relative presentations", Proc. Edinburgh Math. Soc. 35 (1992), 1-39.

[4] W. A. Bogley, and G.Williams, private communication.

[5] D. J. Collins, and J. Huebschmann, "Spherical diagrams and identities among relations", Math. Ann. 261 (1982 ), 155-183.

[6] P. J. Davidson, "On the asphericity of a family of relative group presentations", Internat. J. Algebra Comput. 19 (2009), 159-189.

[7] M. Edjvet, "On the asphericity of one-relator relative presentations", Proc. Roy. Soc. Edinburgh 124A (1994), 713-728.

[8] J. Howie, and V. Metaftsis, "On the asphericity of length five relative group presentations", Proc. London Math. Soc. 82 (2001 ), 173-194. 
[9] V. Metaftsis, "On the asphericity of relative group presentations of arbitrary length", Internat. J. Algebra Comput. 13 (2003 ), 323-339. 